\title{
勇攀高峰, 把基本粒子理论研究搞上去
}

\author{
一一国科学院召开基本粒子理论座谈会
}

在英明领袖华主席关于 “科学要兴旺发 达起来, 要捷报频传”的号召鼓舞下, 中国科 学院于 8 月 1 日至 20 日在安徽黄山召开了 “基本粒子理论座谈会”. 会议在“百家争鸣” 方针指引下，总结交流了国内基本粒子理论 研究的成果; 分析了近年来在国际上高能物 理实验和基本粒子理论研究所取得的成果. 许多单位作了学术报告, 学术空气活跃. 报 告和讨论主要围绕新粒子, 及相互作用和层 子模型的发展等三个专题进行. 此外还进行 了有关规范场与磁单极、量子场论的新发展, 高能强作用过程等方面的报告和讨论. 这些 学术交流反映了我国基本粒子理论研究工作 的新成果, 新水平, 反映了在这一领域中赶超 世界先进水平的新进展.

从 1965 年到 1966 年, 我国基本粒子理 论研究工作者在毛主席的光辉哲学思想的指 导下,提出了“层子模型”理论. 近十年来,由 于林彪和 “四人帮” 的干扰破坏, 研究工作一 度陷于停顿. 在 1972 年敬爱的周总理作了 关于加强基本理论的一系列指示, 在 1973 年 亲自批准召开香山会议以后, 研究工作才重 新开展. 在 “四害” 横行的日子里, 我国的基 本粒子理论工作者尽可能顶住干扰破坏，将 研究工作坚持下来. 四年来我国基本粒子理 论研究工作获得了可喜的成果, 在关于强子 内部波函数和位能之间关系的研究，关于新 粒子的研究, 关于束缚态场论的研究, 以及关 于规范场和磁单极的研究等方面都取得了一 定的进展.

经过讨论,代表们认为: 强子具有内部结 构的观点, 现在已为全世界绝大部份高能物
理研究工作者所接受. 近年来关于轻子和核 子深度非弹性碰撞的实验, 关于弱中性流的 实验, 以及新强子、新轻子的发现, 对基本粒 子理论的发展起了很好的推动作用. 估计充 份发挥现有高能加速器的作用和在七十年代 末新建成的高能加速器投入使用, 将在今后 一个时期促进高能物理实验, 从而推动理论 进一步发展. 估计强子内部结构的理论将会 继续得到发展, 弱相互作用理论将向基本理 论过渡. 更多新粒子的发现预示基本粒子理 论将出现新的生长点.

会议期间，美籍物理学家杨振宁教授由 周培源同志陪同来到黄山. 代表们以很大的 兴趣听取了杨振宁教授所作的三次学术报 告. 在报告中, 杨振宁教授介绍了高能物理 实验最近取得的重要进展, 特别是关于弱相 互作用、新粒子和关于具有分数电荷的粒子 是否存在的实验的结果; 讨论了把纤维丛这 一数学工具引入基本粒子理论的重要前景, 并具体应用于有磁单极存在时规范场的研 究; 讨论了磁单极与荷电粒子形成的系统的 波函数能谱及其物理后果; 报告了关于瞬时 子研究的新成果. 在报告以后, 杨振宁教授 和部份代表进行了座谈.

代表们在认真分析、研究国际、国内基本 粒子理论研究的现状和发展趋势, 初步总结 了我们的经验和教训的基础上, 制订了我国 基本粒子理论研究的发展规划. 在讨论中代 表们对我国基本粒子理论的现状、水平和与 世界先进水平的差距进行了分析, 对我国基 本粒子理论研究的长远目标, 对实现这一目 标分阶段的设想, 对全面安排, 重点保证, 协 
作组织, 具体部署, 以及为实现这一规划所应 采取的各项措施等问题,进行了反复研究,取 得了较为一致的意见。

这次基本粒子座谈会是我国关于基本粒 子理论研究工作的一次重要的会议. 代表们 表示，一定要把党的十一大的公报精神化为
巨大的动力, 最紧密地团结在以华主席为首 的党中央周围,以辩证唯物主义为指导,发扬 不畏艰险, 勇于攀登的精神,以新的面貌和新 的成就迎接全国科学大会的召开，为把我国 建成为伟大的社会主义现代化强国而努力奋 斗.

\section{向癌 症进军的 新起点}

\section{一一全国肪痹会议在北京胜利召开}

在华主席、党中央关怀下,卫生部于 1977 年6月18日至 7 月 2 日在北京召开了全国肿 瘤防治研究工作会议. 会议总结交流了走我 国自己肿㾇防治研究道路所取得的成绩和经 验,制订了肿瘤防治研究规划.

我国劳动人民有着几千年与癌症作斗争 的历史. 但我国关于癌症的现代研究解放后 在毛主席、周总理的亲切关怀下才逐步开展 起来. 1958 年, 特别是无产阶级文化大革命 以来, 肿瘤防治研究工作有了迅速的发展.

几年来, 我国从中央到地方正在建立健 全各级肿瘤防治研究机构; 不少地区, 尤其是 一些农村、工矿高发区防治点和一些城市街 道建立了较完善的防癌网：我国一些重点肿 瘤的农村、工矿高发区防治点继林县食管癌 防治点之后逐步地建立起来, 在抓“三早”(早 期发现、早期诊断、早期治疗)、政“三关”(病 因、诊断、根治) 方面做了不少工作; 各种全 国性和地区性的协作组织不断发展; 由各级 专业人员和赤脚医生、红医工及基层医务人 员组成的防治研究大军茧壮成长; 许多非肿 瘤专业以至非医学的有关科研部门和单位也 参加到肿瘤防治研究工作中来. 流行病学调 查研究工作广泛开展, 进展迅速. 我国仅用 3-5 年的时间即可基本摸清全国肿瘤的发 病情况和分布特点, 为研究发病原因和有计
划地开展防治工作提供了大量科学数据.

我国的肿痹诊断技术几年来有了较大的 改善和提高.一些重点肿瘤如食管癌、肝癌、 鼻咽癌、宫颈癌等的早期诊断水平显著提高, 已能从自觉症状的病人身上发现很早期的肝 癌、食管癌、肺癌等. 通过普查发现的癌病 人，早期瘜比例可达 $80 \%$ 以上，使许多病 人可以获得早期诊断和早期治序. 应用我国 自己创造的食管“拉网”细胞学方法,食管癌 阳性率可达 $90 \%$ ，并可通过分段拉网进行定 位; 改进拉网法, 还提高了贵门癌细胞学诊 断的阳性率, 为贵门癌的早期诊断做出了贡 献; 通过大量实践, 逐步摸清肝癌甲胎蛋白 (AFP) 含量动态变化的一些规律, 建立简便 易行、灵敏度高的测定方法（反向血球凝集 法、晶格参入放射免度火箭自显影法), 使甲 胎蛋白测定成为我国肝癌早期诊断的有效方 法, 并可用于农村大面积普查, 发现了许多早 期无自觉症状的肝㿉病人; 通过不断改进脱 落细胞采集方法, 使鼻咽癌脱落细胞学的阳. 性诊断率达到 $90 \%$ 以上. 几年来,细胞学和 免疫学的一些诊断方法已在我国广泛应用， 在一些高发区正在普及到赤脚医生、红医工 用于普查. 此外, 癌症诊断的不少新技术、新 方法、新仪器我国也已开始应用.

几年来,不少地区,特别是一些防治试点 